\title{
Editorial: High-Energy Density Laboratory Astrophysics 2010
}

\author{
Roberto C. Mancini · Tomasz Plewa • Michael Dopita
}

Received: 6 July 2011 / Accepted: 6 July 2011 / Published online: 6 October 2011

(C) Springer Science+Business Media B.V. 2011

High-Energy Density Laboratory Astrophysics is a fastdeveloping multi-disciplinary field which brings together the work of scientists from universities, laboratories and research institutions in Australia, Canada, China, Czech Republic, Denmark, France, Germany, Italy, Japan, Korea, Netherlands, Poland, Portugal, Russia, Spain, United Kingdom, Uzbekistan, and the United States. The melding of high-energy density plasma laboratory experiments, and their associated theory to problems of direct relevance to astrophysics results in the new discipline of high-energy density laboratory astrophysics. It is therefore appropriate that we here present this Special Issue dedicated to this fastgrowing and important new field.

The laboratory work presented here includes the results of experiments performed with a variety of medium and large-scale high-energy density drivers including the OMEGA and OMEGA-EP lasers, the Jupiter facility lasers, the $\mathrm{Z}$ pulsed-power facility, the LULI2000 laser, and the Magpie and Zebra pulsed-power machines, employing a broad range of radiation and particle based diagnostics. Plans for upcoming experiments at the National Ignition Facility are also discussed.

In addition, this Special Issue presents astronomical observations either directly related to HED experiments already under way or with strong potential for design future experiments. It also covers applications to hydrodynamics and hydrodynamic instabilities, turbulence, radiation- and magneto-hydrodynamics, equation of state, outflows and jets, atomic physics, spectroscopy and radiation processes, stellar atmospheres, and nuclear physics. Clearly, the practice of both Astronomical theory and observation are enhanced by detailed experiments in the laboratory.

We hope that the readers of this special issue will learn much that is new, and will appreciate the exciting new developments that have occurred in the last few years. These demonstrate the impressive fundamental unity of physics over more than twenty four decades of spatial scale and of time.

R.C. Mancini

University of Nevada, Reno, USA

T. Plewa $(\bowtie)$

Florida State University, Tallahassee, USA

e-mail: tplewa@fsu.edu

M. Dopita

Mt. Stromolo Observatory, Canberra, Australia 